\title{
A combined molecular-beam epitaxy and scanning tunneling microscopy system
}

\author{
B. G. Orr, C. W. Snyder, and M. Johnson \\ H. M. Randall Laboratory, University of Michigan, Ann Arbor, Michigan 48109-1120
}

(Received 30 November 1990; accepted for publication 1 March 1991)

\begin{abstract}
A combined molecular-beam epitaxy and scanning tunneling microscopy system has been constructed. The design has been optimized for the study of III-V semiconductors with the goal of examining the surface with both in situ scanning tunneling microscopy (STM) and reflection high-energy electron diffraction (RHEED). Using this system, it is possible to quench the growth and produce real-space images of the surface as it appeared during deposition. Measurements obtained with both RHEED and STM are presented.
\end{abstract}

\section{INTRODUCTION}

Over the past decade molecular-beam epitaxy (MBE) has proven to be a very powerful technique for the production of unique materials. ${ }^{\prime}$ The ability to form films with nearly atomic layer precision has allowed the realization of novel structures with extraordinary enhancement of electronic and optoelectronic properties. To date, the primary technique used to characterize the deposition process has been in situ reflection high-energy electron diffraction (RHEED). Information such as surface diffusion lengths, terrace size, and the symmetry of the surface reconstruction can be obtained. ${ }^{2}$ In addition a statistical picture of the disorder on a surface may be obtained. Although this information has proven to be of tremendous use, RHEED suffers from the shortcomings of any diffraction probe. Data obtained from RHEED is related to the surface reciprocal lattice; therefore, the information is determined from an average over the surface, nonperiodic features are difficult to study, and real-space models are not uniquely determined.

Scanning tunneling microscopy (STM) is a real-space probe with extremely high lateral and vertical resolution. ${ }^{3}$ The strongest features of the technique are: local properties of the surface are studied, defects and nonperiodic features are easily observed, and the data obtained are real-space images of the surface. One of the weaknesses of STM is the inability to scan the surface during growth. Samples generally must be cooled and transferred to a cleaner environment for STM analysis. This procedure allows the surface to evolve, and no longer be representative of the growth front.

Molecular-beam epitaxy when combined with RHEED and STM allows both atomic scale tailoring and atomic resolution imaging of the film surface. The two analytic techniques are highly complementary. The geometry used for RHEED is ideal for monitoring the film during growth whereas STM investigation, as previously mentioned, cannot take place during deposition. With RHEED one can obtain accurate information about the lattice constant, but very little about defects. Whereas with STM, defects are directly observed, but the precision to which the lattice spacing, for example, can be measured is comparatively low.

\section{DESIGN CONSIDERATIONS}

To increase our ability to study MBE film growth, we have developed a system that allows both in situ STM and RHEED analysis of MBE-grown films. The central and novel design criterion for this instrument is the ability to generate real-space images of the sample surface as it appeared during growth. Generally, after growth termination the sample surface continues to evolve due to surface diffusion and remnant flux in the chamber. In an attempt to "freeze" in the growth surface it was desirable to have rapid and complete control over the evaporant fluxes and sample temperature. Therefore, the major design criteria were minimization of vibrations, rapid control of the vapor fluxes (both cation and anion), and responsive sample heating.

Although simple in principle, the equipment used for MBE is generally rather large, complex, and expensive. Perhaps more important, the thermal response of the sample is slow and the vibration levels required for STM operation are not available with many commercial MBE systems. To avoid these problems we chose to design and build a system with both the requirements of STM and $M B E$ in mind. Since this was to be a research machine, the sample size and throughput were not a concern.

\section{IMPLEMENTATION}

GaAs and its alloys are of major technological importance and our studies are centered around their growth. GaAs films are generally grown by producing an overpressure of As (typically $10^{-6}$ Torr) and depositing Ga on the surface at about one quarter that rate. This produces a growth rate of approximately one monolayer per second. The STM cannot be exposed to this growth environment. Therefore a two-section system comprised of growth chamber with RHEED and an analysis chamber equipped with STM was designed. These sections are connected by a welded bellows and gate valve. To reduce vibration the entire system is mounted on a frame suspended from pneumatic dampers. Pumping of the system is done by two 


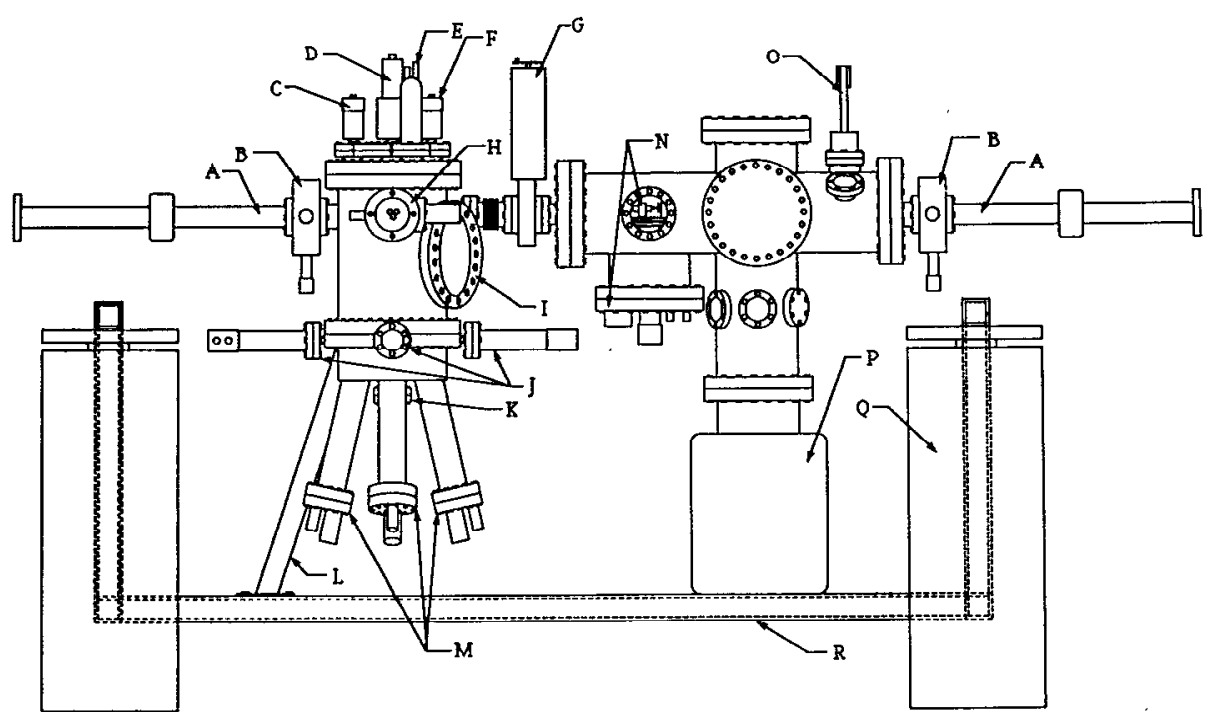

FIG. 1. Overview of the STM-MBE system: (a) linear-rotary feedthrough, (b) $X-Y$ translator, (c) Cu sample shutter, (d) sample manipulator, (e) liquid-nitrogen feedthrough, (f) flux monitor, (g) gate valve, (h) RHEED gun, (i) flange for MBE ion pump (pump not shown), (j) Knudsen shutters, (k) pyrometer port, (1) support for MBE, (m) Knudsen cells, ( $n$ ) STM, (o) sample garage, (p) ion pump, (q) pneumatic legs, and ( $r$ ) frame.

220-1/s triode ion pumps. Samples can be passed from one chamber to another via two magnetically coupled linearrotary motion feedthroughs.

The growth chamber is much smaller than is typically found on a commercial MBE machine. A 10-in. source flange containing four sources, shutters, pyrometer port, and liquid-nitrogen shroud forms the base of the chamber. The top flange contains a liquid-nitrogen feedthrough, shutter control, flux monitor, viewport, sample holder, and heater feedthrough. Into the sides of the chamber protrude the RHEED gun and screen, and ion gauge. An assembly drawing is shown in Fig. 1.

Inside the chamber has been placed a liquid-nitrogencooled $\mathrm{Cu}$ shield and shutter. The shield is placed midway between the top and source flanges. The 6-in. pumping line is bisected by the shield allowing differential pumping between the upper and lower regions of the chamber. A 2-in.diam hole in the center of this plate allows the evaporant flux to reach the sample and flux monitor. This central opening can be sealed by an independently controlled liquid-nitrogen-cooled shutter. When closed there is strong isolation from the remnant As in the lower part of the chamber.

The sample temperature is monitored and feedback stabilized by an infrared pyrometer. As mentioned previously, it is desirable to have a very thermally responsive system. To achieve this we have reduced the sample and heater mass as well as eliminated the heating element. Our samples are rectangular strips of wafer, $1.2 \mathrm{~cm}$ long, 0.8 $\mathrm{cm}$, wide and $0.04 \mathrm{~cm}$ thick. The sample temperature is controlled by passing current through the wafer and resistively heating it. A cooling curve is shown in Fig. 2. It is possible to quench the temperature of the sample from $580^{\circ} \mathrm{C}$ to less than $450^{\circ} \mathrm{C}$ in under $2 \mathrm{~s}$. Using typical values for the surface diffusion constants of $\mathrm{Ga}$ and $\mathrm{As}$ at these temperatures, one finds that the root-mean-squared displacement of a surface atom to be less than $10 \AA$ while the sample is cooling. This ability is unique and cannot be achieved easily by retrofitting a conventional MBE system.

During growth, RHEED patterns are monitored with a charge coupled detector (CCD) camera and video frame grabber. ${ }^{5}$ Intensity oscillations as well as linewidth and lattice constant measurements are recorded simultaneously and displayed in real time. By tracking the evolution of the surface after growth termination we can determine how best to stabilize the growth front surface profile. Shown in Fig. 3 is the intensity of the specularly reflected beam as growth is interrupted. It is apparent that the surface evolution can be quenched, as determined by the constant RHEED intensity, and that the surface does not recover as in a typical MBE situation.

After MBE growth and RHEED analysis the sample is transferred to the STM chamber for imaging. The microscope was built following the design of Demuth et al. ${ }^{6}$ Instead of a screw drive and lever reduction scheme for the course approach we have used a piezoelectric inchworm. ${ }^{(7}$

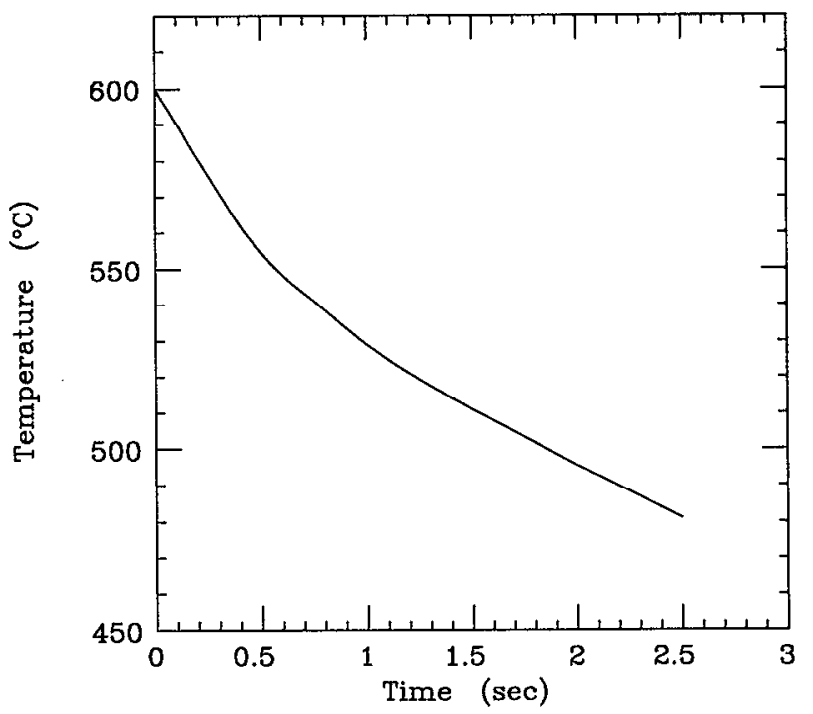

FIG. 2. Graph of the wafer temperature as a function of cooling time for a GaAs sample. The temperature was measured with an infrared pyrometer. 


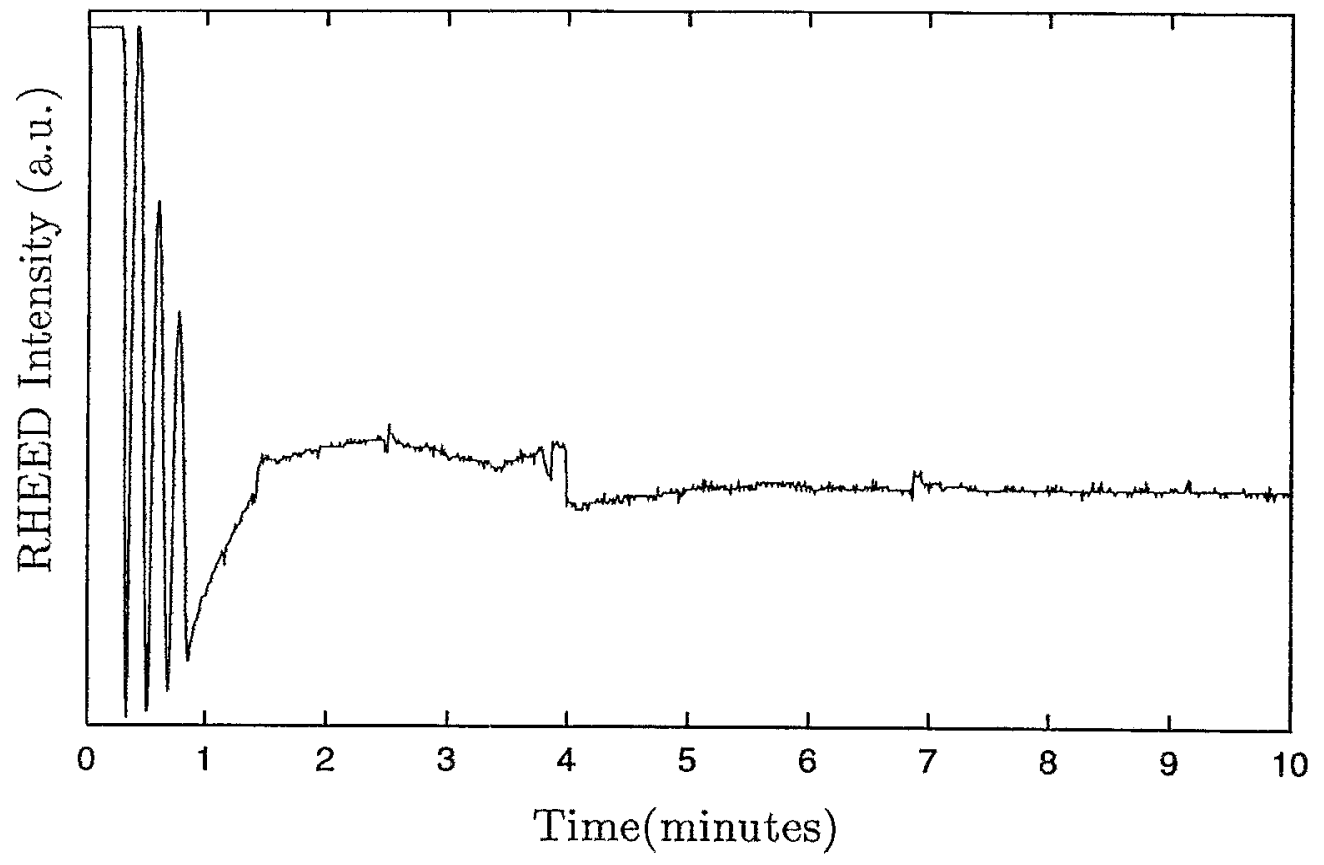

FIG, 3. Graph of the RHEED specular beam intensity as a function of time. At approximately $1 \mathrm{~min}$, growth was interrupted at a RHEED minimum and the sample was allowed to partially recover; then the surface was frozen. Notice the constant intensity level for the scattered beam indicating that the surface evolution can be inhibited. (The offset at $4 \mathrm{~min}$ is an artifact caused by altering the heater current.)
Vibration isolation is accomplished with spring suspension ( $2 \mathrm{~Hz}$ resonance) and plates separated by elastomer spacers. The tip is mounted on a crcss machined from piezoelectric material. This scanner provides the lateral movement of the tip. A stick piezo below the cross scanner is used to control motion perpendicular to the sample surface. The microscope electronics and software were purchased commercially. ${ }^{8}$ Figure 4 shows a STM image of a GaAs buffer layer that has been allowed to recover. Rows generated by the $2 \times 4$ reconstruction are clearly visible. To further demonstrate our ability to quench the growth surface we have examined GaAs buffer layers where the surface recovery has been inhibited. The surface is very much rougher with islands approximately $50 \AA$ in diameter and $10 \AA$ high. Annealing studies and. Monte Carlo simulation

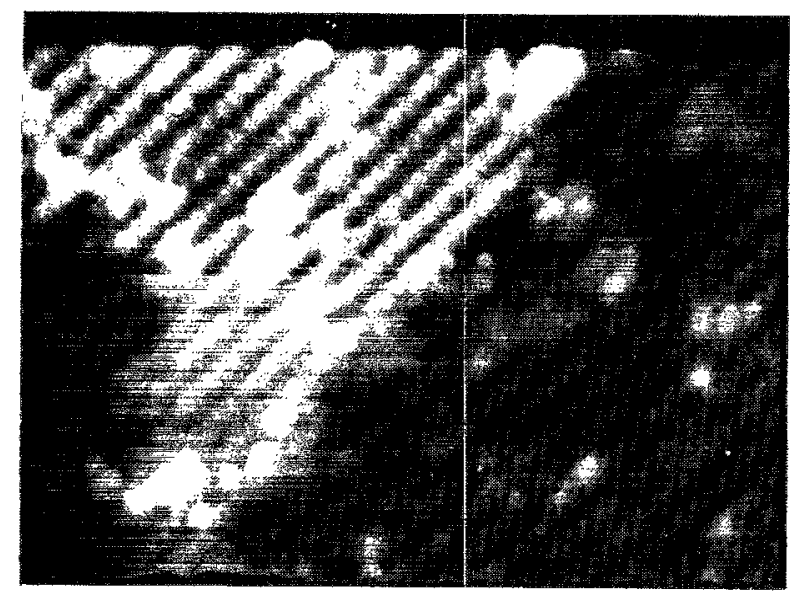

FIG. 4. STM image of a GaAs buffer layer. The image size is $400 \times 280 \AA \hat{\text {. }}$ In the center of the image is a monolayer step. are presently underway to explain the decay of the RHEED oscillations and observed roughness.

We have also studied various phases of strained layer growth with this instrument. ${ }^{9}$ The deposition process can be monitored as the film passes through the critical thickness where dislocations are expected to form. Figure 5 shows an image of an $\mathrm{In}_{0.4} \mathrm{Ga}_{0.6}$ As film after $12 \AA$ (before critical thickness) have been deposited on GaAs. Similar studies have shown that by quenching we are able to inhibit the formation of facets on this film after it exceeds critical thickness. With annealing, the facets are recovered. ${ }^{10}$ If only examination of the slowly cooled film could be achieved then the true state of the growing film would remain unknown. In general, to make a proper comparison between STM and RHEED measurements the surface

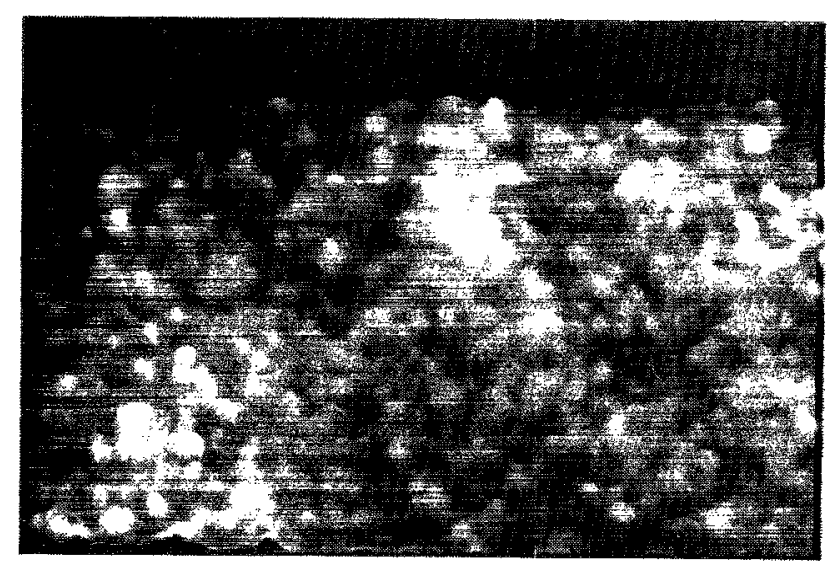

FIG. 5. Image of a 12 -A -thick $\operatorname{In}_{0.4} \mathrm{Ga}_{0.6} \mathrm{As}$ film. The image size is $500 \times 880 \AA$ A. The surface is covered with islands roughly $50 \AA$ in diameter and $6 \AA$ high. 
must be imaged in the unrecovered state. The key to understanding the growth of epitaxial films is finding the structure and evolution of the surface as it exists during deposition. This instrument has demonstrated that capability.

In summary, we have constructed a combined MBE and STM system. By optimizing the system design we have been able to study GaAs and its alloys. The system allows surfaces to be controlled with monolayer precision and imaged with atomic resolution. We have demonstrated that by a combination of rapid thermal quenching and shrouding we have been able to study the surface as it appeared during growth. The combination of these techniques significantly enhances our ability to study epitaxial film growth.

\section{ACKNOWLEDGMENTS}

We would like to thank D. Barlett for development of the RHEED CCD detection system and R. Sears for assis- tance with data processing. In addition, we would like to thank RHK Technology for the donation of equipment. This work has been supported by Grants: No. DAAL0388-G-0058, No. N00014-89-J-1519, No. NSF/DMR8857828, and the Sloan Foundation.

${ }^{1}$ E. Parker, Ed., The Technology and Physics of Molecular Beam Epitaxy (Plenum, New York, 1985).

${ }^{2}$ P. K. Larsen and P. J. Dobson, Eds., Reflection High-Energy Electron Diffraction and Reflection Electron Imaging of Surfaces, edited by $\mathbf{P}$.

K. Larsen and P. J. Dobson, (Plenum, New York, 1988).

${ }^{3}$ G. Binnig, H. Rohrer, Ch. Gerber, and E. Wcibel, Phys. Rev. Lett. 50, 120 (1983); IBM J. Res. Dev. 4/5, 30 (1986).

${ }^{4}$ EPI Systems, St. Paul, MN 55101.

${ }^{5}$ D. Barlett, C. W. Snyder, B. G. Orr, and R. Clarke, Rev. Sci. Instrum. 62, 1263 (1991).

${ }^{6}$ J. E. Demuth, R. J. Hamers, R. M. Tromp, and M. E. Welland, J. Vac. Sci. Technol. A 4, 1320 (1986).

${ }^{7}$ Burleigh Instruments, Fishers, NY 14453.

${ }^{8}$ RHK Technology, Rochester Hills, MI 48063.

${ }^{9}$ C. W. Snyder, D. Barlett, B. G. Orr, P. K. Bhattacharya, and J. Singh, J. Vac. Sci. Technol. (to be published).

${ }^{10}$ B. G. Orr, C. W. Snyder, and M. Johnson (unpublished). 